\title{
Treatment of band keratopathy by excimer laser phototherapeutic keratectomy: surgical techniques and long term follow up
}

\author{
D P S O’Brart, D S Gartry, C P Lohmann, A L Patmore, M G Kerr Muir, J Marshall
}

\begin{abstract}
A series of 122 eyes with band keratopathy was treated by excimer laser phototherapeutic keratectomy (PTK), with a mean follow up of over 12.3 months (range 3 to 60 months). A single photoablation zone was used to remove the opacity over the visual axis in smooth surfaced band deposition. In eyes with reduced vision, an improvement was reported in $88 \%$ and in a series of 66 eyes mean Snellen visual acuity increased significantly $(p<0.05$, $t=2 \cdot 27$ ). A reduction in glare was reported in $88 \%$ and in a series of 17 patients, visual contrast sensitivity $(p<0.01)$ and measurements of disability glare $(\mathbf{p}<0.01)$ improved postoperatively. The mean hyperopic shift in 32 eyes at 6 months was $1.4 \mathrm{D}$ (range 0-4.25 D). Multiple overlapping ablation zones, with mechanical debulking of large calcium plaques, were used to smooth the irregular corneal surface in eyes with rough bands. Ocular discomfort was improved in $95 \%$. Band keratopathy recurred in nine eyes $(8 \%)$ within 2 to 30 months (mean 12 months) of surgery, with silicone oil responsible in five eyes. Reablation was necessary in three eyes and performed successfully in all cases. Excimer laser PTK is a safe and effective outpatient treatment for band keratopathy.

(Brf Ophthalmol 1993; 77: 702-708)
\end{abstract}

Band shaped keratopathy was first described by Dixon in $1848^{1}$ and subsequently by his associate Bowman in $1849 .{ }^{2}$ It is a degenerative condition, characterised by the slow development of a grey white opacity of the superficial cornea, usually in the interpalpebral zone.

Patients may complain of glare and disturbed vision when the pathology extends over the visual axis. Provided the epithelial surface remains intact such eyes are comfortable, but once it is breached there is considerable ocular irritation.

The aim of surgical treatment is to remove the opaque calcium deposits and/or restore the normal smooth corneal surface. Chelating agents - for example, ethylenediaminetetra-acetic acid (EDTA), ${ }^{34}$ and superficial keratectomy have been advocated. ${ }^{56}$ However, the calcium deposition may be resistant to removal with EDTA and mechanical keratectomy may result in an irregular surface.

Clinical excimer lasers have become available since the mid 1980s and offer a new approach to the treatment of superficial corneal pathologies.? The advantages of such lasers derive from two unique characteristics. The first is the ability to remove corneal tissue with extreme precision and minimal adjacent tissue damage..$^{8-11}$ The second is large beam cross sections, typically several millimetres in diameter, which allow simultaneous treatment of wide areas.

Excimer lasers have been used to remove superficial opacities from the corneal visual axis and to smooth irregular corneal surfaces. ${ }^{72-15}$ The former results in an improvement in vision and a reduction in glare (12-14 $^{12}$ and the latter produces increased ocular comfort ${ }^{7}$ or allows the subsequent use of contact lenses. ${ }^{14} 15$

The results of excimer laser phototherapeutic keratectomy (PTK) in previous studies include a maximum follow up of 28 months, with only small numbers treated for band keratopathies. We present a series of 97 patients (122 eyes) with band keratopathy treated by PTK, with a maximum follow up of 60 months. We also report on one pathological specimen, obtained 12 months after laser surgery.

\section{Materials and methods}

\section{EXCIMER LASER}

A Summit Technology UV200 excimer laser was used with a spectral emission at $193 \mathrm{~nm}$. The pulse energy resulted in a radiant exposure of $180 \mathrm{~mJ} / \mathrm{cm}^{2}$ and the pulse frequency was fixed at $10 \mathrm{~Hz}$. The beam configuration was circular in cross section, with fixed diameters, selected to be between $1 \cdot 0-5 \cdot 0 \mathrm{~mm}$.

\section{SUBJECTS}

There were 97 patients (122 eyes) treated for band keratopathy. Although there was a large morphological spectrum these patients could be separated into two broad groups: 'smooth' and 'rough'. In smooth surfaced band deposition the epithelium was intact with the calcified material lying at the level of Bowman's layer. These eyes were comfortable and usually had good visual potential. In rough band keratopathy the epithelium was unstable over calcified plaques. These were often painful, blind eyes. The aetiology of all cases is shown in Table 1 .

Smooth bands were treated in 78 eyes of 56 patients. The average age was 66 years and the mean follow up was 10 months (range 3-54 months). In 18 cases the follow up was over 12 months, while in nine it was over 2 years. Preoperatively, reduced vision was reported in 68 eyes and 48 had glare. Three eyes were treated to improve the peroperative view for cataract surgery.

Rough band keratopathies were treated in 44 
Table 1 Aetiology for band keratopathy in 97 patients

\begin{tabular}{lc}
\hline Aetiological condition & $\begin{array}{c}\text { Number of } \\
\text { patients }\end{array}$ \\
\hline (i) Aetiology for smooth surfaced band deposition in & 56 patients \\
Primary (idiopathic) & 17 \\
Associated with glaucoma & 15 \\
Still's/childhood uveitis & 7 \\
Silicone oil & 6 \\
Sarcoid/hypercalcaemia & $3 \dagger$ \\
Other & 8 \\
(ii) Aetiology for rough band keratopathy in 41 patients & 8 \\
Trauma (mainly penetrating) & 7 \\
Herpes zoster & 4 \\
Herpes simplex keratitis & 4 \\
Silicone oil & 3 \\
Glaucoma (blind eyes) & 2 \\
Vernal plaques & $2 \dagger$ \\
Sarcoid/hypercalcaemia & 2 \\
Uveitis (blind eyes) & 9 \\
Other & \\
\hline
\end{tabular}

*Two patients had smooth calcium deposition in one eye and a rough band in the other.

tIn one patient rough calcified plaques eroded through the tIn one patient rough calcified plaques eroded through the
peripheral corneal epithelium, 18 months after successful peripheral corneal epithelium,
treatment for a smooth band.

eyes of 41 patients. The average age was 56 years and the mean follow up was $16 \cdot 3$ months (range 3-60 months). Follow up was greater than 12 months in 21 cases and 24 months in 13. Preoperatively ocular discomfort was reported in 37 eyes, reduced vision in nine, and four patients were treated to allow cosmetic contact lenses to be worn.

\section{ASSESSMENT AND EXAMINATION}

Patients were fully counselled before surgery and a detailed ocular examination performed. Corneal topography was examined in selected patients using a photokeratoscope (TMS-1 Topographic Modelling System, Computed Anatomy). Where possible, a full refraction was performed. In 17 patients with smooth band keratopathy and Snellen visual acuities of $6 / 18$ or better visual contrast sensitivity and disability glare were measured preoperatively and 1 month after surgery using a specially designed computer program, which has been previously described. ${ }^{17}$

\section{THE PROCEDURE}

A local anaesthetic (amethocaine hydrochloride $1 \%$ ) was instilled. Where possible the patient was



Fig IA trained to fixate on the target light for the predicted duration of the procedure. In bland eyes some patients were able to maintain fixation using the fellow sighted eye. The noise associated with the laser was demonstrated to the patients and they were warned to expect a smell of burning during treatment. A lid speculum was inserted and the relevant procedure performed.

Postoperatively a mydriatic (homatropine 2\%) and an antibiotic ointment (chloramphenicol $1 \%)$ were instilled and the eye padded for 24 hours. Oral analgesics were prescribed for the first 48 hours and chloramphenicol $0.5 \%$ eye drops were administered four times a day for 10 days. The majority of patients were followed up at 1 week and $1,3,6,9$, and 12 months and annually thereafter.

\section{SURGICAL TECHNIQUES}

\section{Smooth band keratopathy}

The axial opacification was removed using a single large ablation $(4 \cdot 0-5 \cdot 0 \mathrm{~mm})$ zone. In contrast to PRK the epithelium was not mechanically debrided. The intact epithelial surface acted as a 'template' for the ablation procedure and no masking agents were required. All laser pulses resulted in fluorescence in the target tissue but a brighter fluorescence occurred during ablation of the calcified deposits and the end point was determined by cessation of this bright fluorescence. Usually less than 300 pulses were required to ablate through both the epithelium and the calcium to leave a clear circular 'window' over the visual axis (Fig 1).

\section{Rough band keratopathy}

In eyes with a poor visual prognosis treatment could be performed without the need to consider the refractive changes that might be induced. Multiple overlapping ablation zones were used to smooth the irregular corneal surface. Any large calcified plaques were removed with a scalpel before PTK. These had a lower ablation rate per pulse than the surrounding corneal tissue and attempts to photoablate them directly may have resulted in deep gutters being excised in the cornea around their edges. The troughs created in the stroma by the removal of the plaques were



Fig $1 B$

Figure 1 Smooth surfaced band keratopathy. (A) The preoperative appearance. (B) The appearance 18 months after excimer ablation of a single axial zone. The increased clarity of the ablated area is evident. The axial zone remains clear 36 months after surgery. 


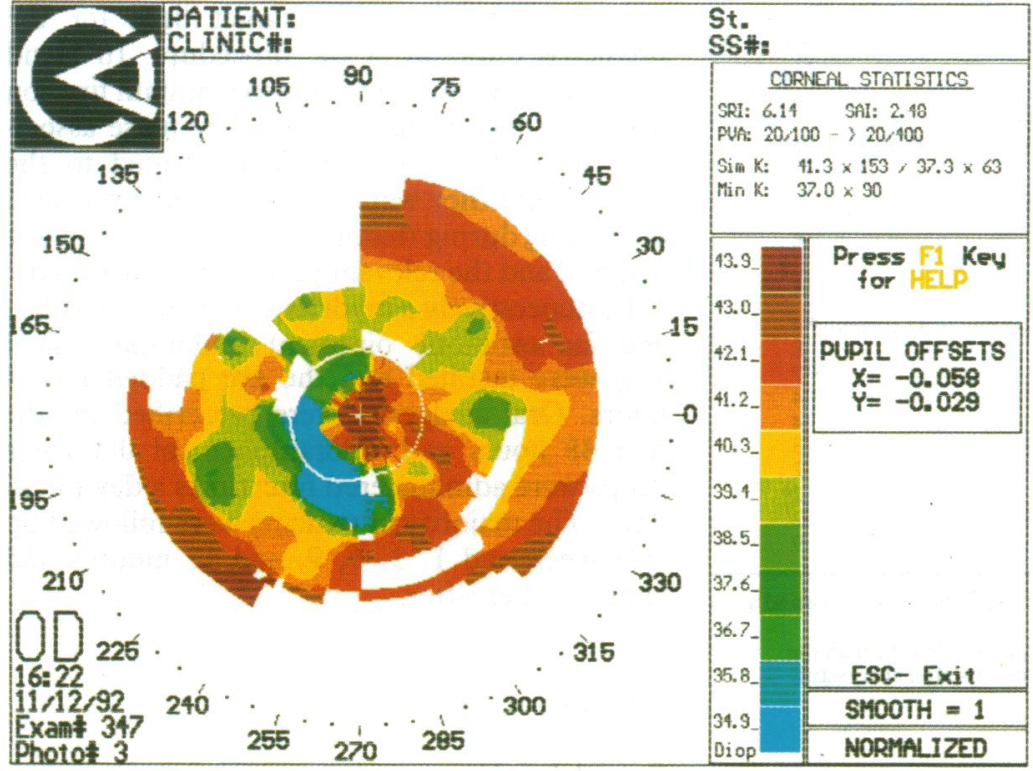

Fig $2 A$

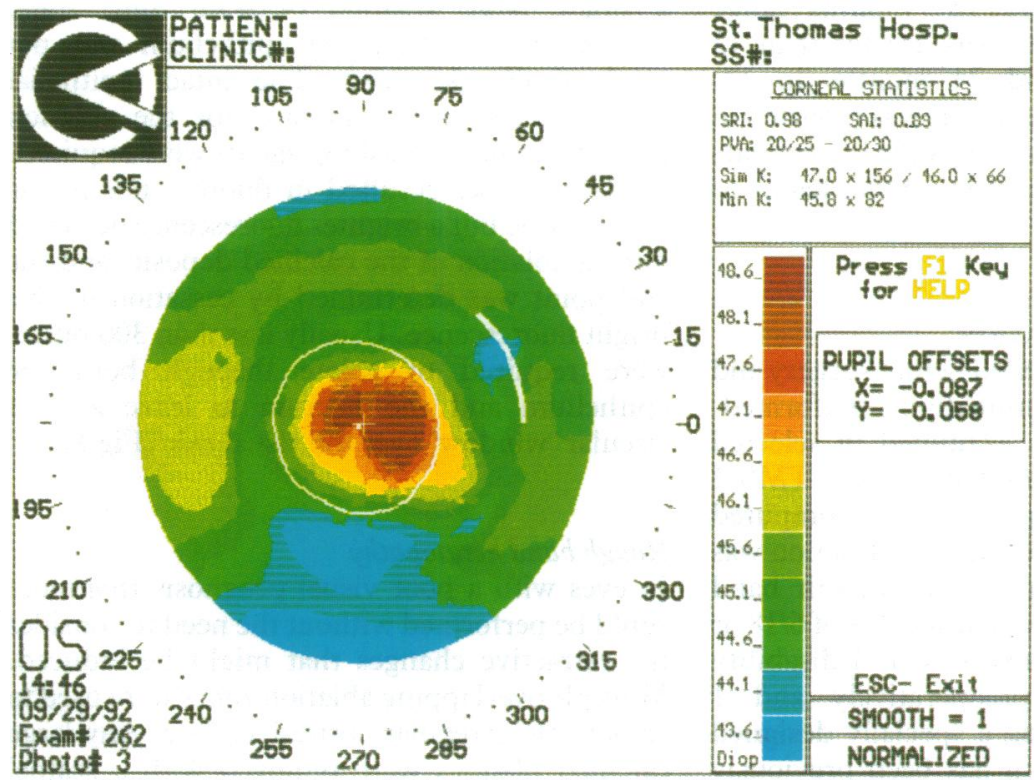

Fig $2 B$

Figure 2 Photokeratoscopic images, computed anatomy, TMS-1, after PTK for smooth band keratopathy. (A) Appearance 34 months after treatment with multiple overlapping ablation zones. Despite adequate clearance of the axial band, the patient complains of multiple images in this eye and best spectacle corrected Snellen visual acuity fell by two lines postoperatively. (B) Appearance 3 months after ablation with a $5.00 \mathrm{~mm}$ axial zone. This patient has a visual acuity of $6 / 9+$ with best spectacle correction and has noted a marked improvement in glare disability postoperatively. There was a slight hyperopic shift of $0.75 \mathrm{D}$ at 3 months.

filled with a masking fluid to allow the surgeon to create a smooth surface for epithelial coverage. As in previous studies ${ }^{7}$ hydroxypropylmethylcellulose (HPMC) 1\% was used since it was easy to apply and remove and filled the declivities between surface irregularities. The fluid was delivered to the cornea using microcollibri forceps. This was dipped into the HPMC, the surface tension of the fluid drawing a small amount between the closed ends of the forceps. Fluid could then be accurately 'painted' onto the cornea. During photoablation the HPMC fluoresced bright blue with the stromal peaks appearing dark blue black. This inferred a three dimensional geometry of the stroma undergoing ablation and allowed the surgeon to monitor the process. During the smoothing procedure it was often useful to slightly move the patient's eye, by the surgeon gently moving the patient's head. With successive pulses falling on slightly different areas of the target a polishing effect was produced. Perioperative slit-lamp examination was useful in assessing the ablation depth and the regularity of the treated area.

In eyes with visual potential a two stage procedure was adopted. Firstly, the axial zone was treated with a single large ablation zone and then overlapping multiple zones used to smooth any irregularities in the periphery. Care was taken to avoid incursion of the multiple peripheral zones on the central region so as not to induce irregular astigmatism (Fig $2 \mathrm{~A})$.

\section{HISTOPATHOLOGY}

A single corneal specimen was obtained from an 18-year-old patient who underwent a penetrating keratoplasty procedure for corneal decompensation secondary to silicone oil. Her vision improved after excimer laser PTK for smooth band keratopathy 12 months previously.

The corneal button was immersed in $2.5 \%$ glutaraldehyde buffered in $1 \mathrm{M}$ sodium carcodylate with $10 \mathrm{~g} / \mathrm{l}$ calcium chloride at a final $\mathrm{pH}$ of $7 \cdot 4$. The specimen was postfixed for 1 hour in $2 \%$ osmium tetroxide buffered in $0.1 \mathrm{M}$ sodium cacodylate, dehydrated in alcohol, and then embedded in Araldite (CY212) via epoxypropane. Sections were cut at $1 \mu \mathrm{m}$ on glass knives and examined by light microscopy.

\section{STATISTICAL METHODS AND DATA ANALYSIS}

Mean visual acuities were computed by averaging the decimal equivalent of Snellen visual acuity with best spectacle correction, and paired $t$ tests were used to assess changes from the preoperative to the postoperative value. Wilcoxon's rank sum tests were used to assess changes in visual contrast sensitivity and glare disability measurements pre and postoperatively as the data were not normally distributed. There were 24 patients who had both eyes treated. The pathology was asymmetrical in these cases and the eyes were assumed to be independent for statistical analysis. Results with $\mathrm{p}<0.05$ were considered statistically significant.

\section{Results}

SMOOTH BAND KERATOPATHY

There were 78 eyes treated for smooth band keratopathy (Fig 1). The average number of pulses required to clear the visual axis in 58

Table 2 Computerised visual contrast sensitivity and glare disability measurements in 17 patients with smooth band keratopathy. All patients had best corrected Snellen visual acuities of $6 / 18$ or better. All measurements were made with the patients' best spectacle correction for near placed in trial frames

\begin{tabular}{lll}
\hline & Glare source off & Glare source on \\
\hline Av preop value & $21 \cdot 8$ & $57 \cdot 8$ \\
Preop range & $0 \cdot 1-67 \cdot 8$ & $32 \cdot 0-80 \cdot 0$ \\
Av postop value & $7 \cdot 1$ & $35 \cdot 0$ \\
Postop range & $0 \cdot 5-19 \cdot 3$ & $17 \cdot 6-48 \cdot 1$ \\
\hline
\end{tabular}




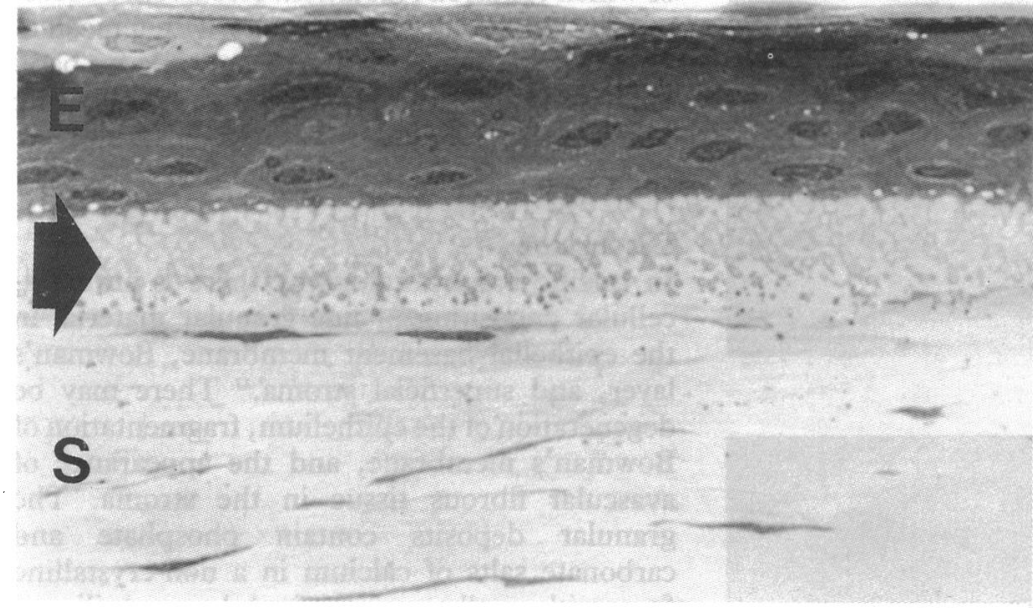

Fig $3 A$

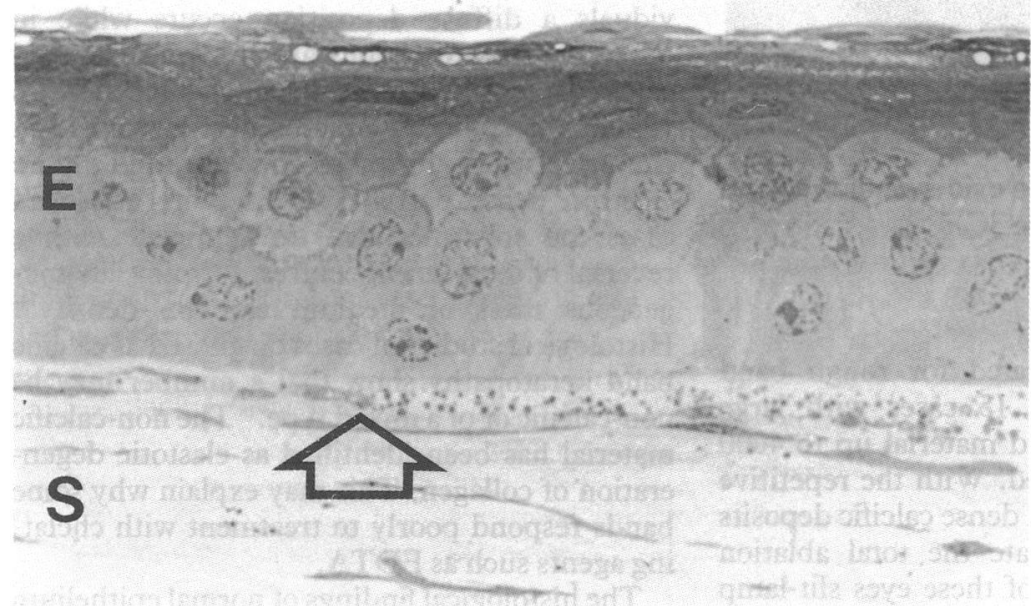

Fig $3 B$

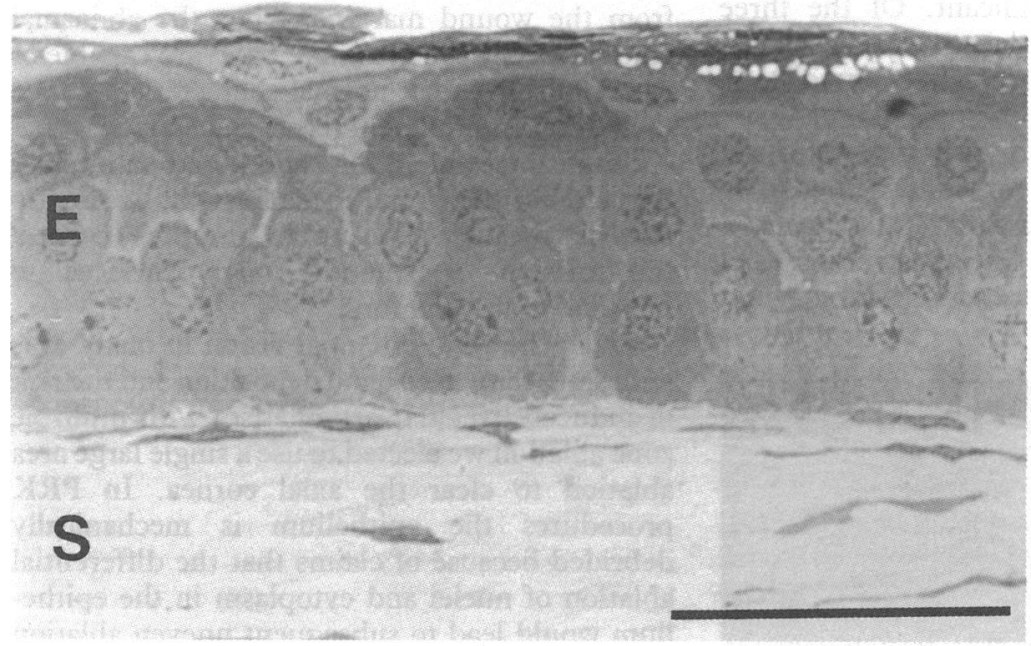

Fic 3C

Figure 3 Light micrographs of samples from the central cornea of an 18-year-old female 12 months after PTK for smooth band keratopathy. $E=$ epithelium, closed arrow=Bowman's layer, $S=$ stroma. The bar marker is $50 \mu \mathrm{m}$. (A) Non-treated area showing Bowman's layer filled with diffuse band deposit. $(B)$ Transition zone between treated and non-treated regions (open arrow). (C) Appearance within the central ablated area. procedures was 268 (range 181-350). All patients had discomfort until epithelial closure which usually occurred within 48 hours. Postoperative recovery was rapid and good visual performance returned within a few days.

In the 68 eyes with reduced vision preoperatively an improvement was noted in $88 \%$. Mean Snellen acuity in 66 eyes improved significantly $(\mathrm{p}<0.05, t=2 \cdot 27)$. In the 48 eyes with symptomatic glare preoperatively an improvement was reported in $88 \%$. In a series of 17 patients in whom detailed measurements of visual performance were made visual contrast sensitivity $(\mathrm{p}<0.01)$ and disability glare $(\mathrm{p}<0.01)$ improved at 1 month postoperatively (Table 2 ).

Of the three eyes treated to facilitate cataract surgery, the improvement in corneal clarity enabled all to have uncomplicated extracapsular procedures.

Complications included recurrent erosions (two episodes) in one patient which settled after 3 months and with no symptoms for over 2 years. A reduction in postoperative Snellen acuity occurred in the first two eyes treated despite adequate removal of the band. In these cases multiple overlapping ablation zones were used over the central cornea and resulted in irregular astigmatism (Fig 2A). In all subsequent procedures a single axial zone was used which produced a more regular optical surface (Fig 2B). Postoperative subepithelial opacity was minimal and the majority of patients had clear axial corneas at 6 months. All eyes had a hyperopic shift and the mean in 32 eyes at 6 months was 1.4 dioptres (range $0-4 \cdot 25 \mathrm{D}$ ). Six eyes $(19 \%)$ had hyperopic shifts over 2.0 D.

\section{HISTOPATHOLOGY}

The histological appearances of ablated and nonablated areas of a keratoplasty button from an 18 year-old female are shown in Figure 3. In the non-treated area the epithelium had six cell layers but appeared attenuated with an overall thickness of $26 \mu \mathrm{m}$. All cell layers appeared abnormal with focal aggregations of dense nuclear stain. There was no clear columnar basal layer and all cells had a wing-like cell appearance. The basal surface of the basal layer was undulant. The band material was typically spherical in form with a diameter of about $1 \mu \mathrm{m}$. It extended throughout Bowman's layer with an overall thickness of $13 \mu \mathrm{m}$ but became less dense towards the stromal surface. The superficial stroma appeared normal with no abnormalities in adjacent keratocytes (Fig 3A).

At the interface between ablated and nonablated area the epithelial thickness increased to $40 \mu \mathrm{m}$ and the cellular appearance became more normal. The basal cells had a linear basal border and the underlying inclusions showed a gradual transition zone (Fig 3B).

Within the treated area the epithelial thickness was $46 \mu \mathrm{m}$ and all six cell layers showed normal anatomical features. The basal cells had a linear basal border although the linearity was not comparable to that found over normal Bowman's layer. The only abnormalities present in this region were the absence of Bowman's layer and a slight increase in the population density of 
Figure 4 Rough band keratopathy. (A) The preoperative appearance of a patient with extensive a grossly irregular ocular surface. (B) The appearance 24 months after treatment of the central cornea. The majority of the calcium was ablated in this region, leaving a smooth surface. Peripheral deposits were not completely removed and some minimal recurrence of band keratopathy has resulted in episodes of minor irritation. The eye has been comfortable for 5 years. calcium deposition producing

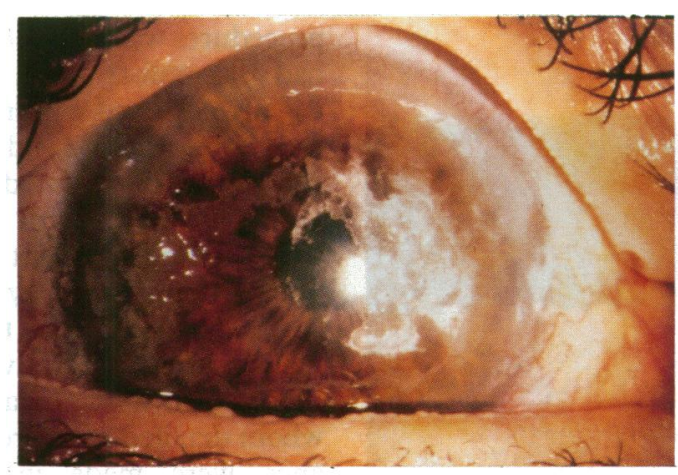

Fig 4A

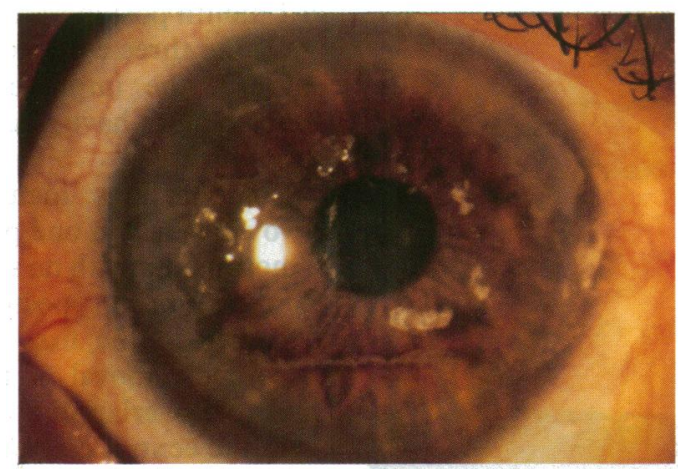

Fig $4 B$

superficial keratocytes. No evidence of the band material could be seen (Fig 3C).

\section{ROUGH BAND KERATOPATHY}

There were 44 eyes treated for rough band keratopathy (Fig 4). In 15 cases with large amounts of dense calcified material up to 4000 laser pulses were delivered. With the repetitive use of masking agents and dense calcific deposits it was difficult to evaluate the total ablation depth although in some of these eyes slit-lamp examination indicated that the excision extended into the posterior half of the stroma.

Improved comfort was reported in $95 \%$ with preoperative ocular irritation. The mean Snellen acuity in 43 eyes improved, but the difference was not statistically significant. Of the three patients who were unable to wear cosmetic contact lenses all were successfully fitted postoperatively.

Complications included recurrent erosions (two episodes) in one patient which settled after 6 weeks. In one eye treated with multiple zones a reduction in postoperative Snellen acuity (6/12 to $6 / 18$ ) resulted from irregular astigmatism.

Figure 5

Macrophotograph showing recurrence of band keratopathy 6 months after treatment for smooth band keratopathy in a patient with silicone oil in the anterior chamber. There is a granular deposition of band material within the treated axial zone surrounded by a densely opaque peripheral area of untreated calcium deposition. The arrow points to the edge of the previously treated zone. Reablation has been successfully performed.
RECURRENCE OF BAND KERATOPATHY

There were nine cases of recurrence (Fig 5) three of which required reablation. Recurrence occurred 2 to 30 months after surgery with a mean of 12 months. Silicone oil was responsible for band keratopathy in five of these eyes, three were smooth-surfaced, and two were rough bands.

\section{Discussion}

In calcific band keratopathy there is an extracellular deposition of fine granular material in the epithelial basement membrane, Bowman's layer, and superficial stroma. ${ }^{17}$ There may be degeneration of the epithelium, fragmentation of Bowman's membrane, and the appearance of avascular fibrous tissue in the stroma. The granular deposits contain phosphate and carbonate salts of calcium in a non-crystalline form with small amounts of sulphur and silicone in advanced cases. ${ }^{5}$ The origin of the calcium salts and the mechanisms which lead to their deposition are obscure but are associated with a number of diverse ocular and systemic disorders (Table 1). It is also unclear why in some individuals a diffuse deposition occurs while in others focal aggregations are apparent. In mild cases the calcium salts form small spherules which on electron microscope examination characteristically have a lucent centre and a more electron dense periphery. ${ }^{18}$ In more advanced cases the spherules tend to aggregate with a reversal of their lucent centres, forming a homogeneous mass of medium electron density. ${ }^{18}$ Histological studies of cases diagnosed as calcific band keratopathy show that a number may be non-calcific or of a mixed type. ${ }^{18}$ The non-calcific material has been identified as elastotic degeneration of collagen. This may explain why some bands respond poorly to treatment with chelating agents such as EDTA.

The histological findings of normal epithelium over the ablated area with abnormal epithelium outside this region (Fig 3 ) suggest that the presence of band material adversely affects epithelial maturation and function. Epithelium heals by migration and then proliferation of cells from the wound margins. Thus the abnormal epithelial cells overlying the band are capable of re-establishing their normal characteristics once their basal environment has been changed.

Surgical treatment for band keratopathy does not address the causal mechanisms but aims to remove opaque deposits to improve corneal transmission or smooth rough surfaces to improve ocular comfort.

Given the good potential vision in many eyes with smooth surface band deposition and the risk of induced irregular astigmatism with multiple zone ablation we elected to use a single large area ablation to clear the axial cornea. In PRK procedures the epithelium is mechanically debrided because of claims that the differential ablation of nuclei and cytoplasm in the epithelium would lead to subsequent uneven ablation of the stroma. In calcific band keratopathy the calcium aggregations have a lower ablation rate than surrounding corneal tissue and an individual particle size in the order of $1 \mu \mathrm{m}$. The distribution of the band is not uniform, typically 
becoming less dense temporally and inferiorly. Although the pathology in smooth bands is restricted to a thin deposit on the outer aspect of Bowman's layer, with little or no stromal involvement, the differential ablation rate between the band and surrounding corneal tissue still might be expected to produce surface irregularities across the ablation zone. However, these were not detectable on gross observations and after re-epithelialisation patients had a regular optical surface (Fig 2B). No patients treated with single axial zones had a reduction in postoperative visual acuity due to irregular astigmatism. From these observations the potential surface undulations produced by biological materials with marginally different ablation rates appear to be minimal and perhaps manual debridement of the epithelium before PRK is unnecessary.

Differential fluorescence between the band and the stroma gives a precise end point for laser exposure and normally less than 300 pulses were required to ablate through the intact epithelium and the band. Concern has been expressed regarding the potential hazards associated with tissue fluorescence. Although $90 \%$ of photons at $193 \mathrm{~nm}$ will be absorbed within the first micrometre of the tissue surface the longer wavelengths of this secondary fluorescence have greater penetration and may be more biologically active. A recent study has shown that hundreds of thousands of pulses would be required to give rise to sufficient secondary fluorescence to induce either a photokeratitis or cataract. ${ }^{19}$

If the laser pulses were falling on a normal corneal surface with an intact epithelium 300 pulses would result in a maximum total ablation depth of $80 \mu \mathrm{m}$. Therefore in many cases the excision may not extend beyond Bowman's membrane. By restricting the depth of ablation it is possible to avoid an excessive hyperopic shift and possibly reduce the propensity for corneal haze. The mean hyperopic shift in 32 eyes at 6 months was less than $1.50 \mathrm{D}$. Postoperative corneal haze was not a problem in our patients and when present was insignificant compared with the preoperative opacity.

After treatment for smooth band keratopathy almost $90 \%$ of patients reported an improvement in vision and glare and there was a significant increase in Snellen acuity $(\mathrm{p}<0.05)$. It is important to note that even patients with good preoperative visual acuity may derive considerable benefit from treatment due to an improvement in glare (Table 2).

In rough band keratopathies large calcium plaques were usually removed mechanically before photoablation. This has three advantages over direct ablation: firstly, the total procedure time was reduced; secondly, the constant application of a shielding agent was found to be unnecessary; and thirdly, as the differential ablation rate of masking fluid to corneal tissue was less than that of calcium to corneal tissue, it was easier to smooth the ocular surface once the major calcium plaques had been removed. Once the calcium plaques had been removed the keratectomy bed was ablated smooth using a masking agent. The important properties of a masking fluid are its ablation rate at $193 \mathrm{~nm}$, its viscosity, and surface tension. HMPC $1 \%$ was ideal for the majority of our cases while polyvinyl alcohol and 2\% HPMC were too viscous and did not have good surface contour following properties. In the choice of any masking agent care must be taken that it does not contain any fluorophores and that on photoablation no toxic substances are produced.

There were six patients with rough calcified surfaces who had already undergone one or more surgical superficial keratectomy procedures. All preferred treatment with the excimer laser, because the speed and comfort of the procedure, a more rapid recovery time and less postoperative discomfort.

In previous studies it has been suggested that only pathology within the anterior $100 \mu \mathrm{m}$ of the stroma should be treated. ${ }^{14}$ In a number of our patients with blind eyes and rough calcified surfaces, the ablation was continued much deeper than $100 \mu \mathrm{m}$ into the corneal stroma with up to 4000 laser pulses being used in individual cases. These eyes have healed satisfactorily and with up to 60 months' follow up, corneal melting, vascularisation, or ectatic changes have not occurred.

Rough bands induce a foreign body sensation and it is important to examine the upper tarsal conjunctiva before surgery. Patients with ocular pain other than a foreign body sensation with no inflammatory signs on the upper tarsus are unlikely to derive benefit from treatment and other explanations for the pain should be sought.

Calcific band keratopathy is a disorder associated with a wide variety of ocular and systemic diseases (Table 1). In eyes with rough calcified surface there was often severe underlying pathology, such as penetrating trauma, previous herpetic disease, severe uveitis, and end stage glaucoma. In patients with smooth bands no predisposing cause could be found in $30 \%$. They had no family history of ocular disease, had otherwise healthy eyes and normal serum calcium levels. There was a significant number (28\%) with glaucoma. Band keratopathy has been reported following long term pilocarpine treatment ${ }^{20}$ and is thought to result from the presence of phenylmercuric nitrate preservatives. Most of our patients had never used pilocarpine eyedrops, being adequately managed with topical $\beta$ blockers. Phenylmercuric nitrate has not been used as a preservative in pilocarpine since the mid 1970s and the majority of the patients were diagnosed after this time. Some adrenaline eyedrop preparations contain mercuryl preservatives but only two patients were using these. The nature of the pathogenesis of band keratopathy in these cases is unclear but there we could not find a relation with phenylmercuric nitrate.

Recurrence occurred in five of the 10 eyes in our series with band keratopathy secondary to silicone oil. Oil was present in the anterior chamber in contact with the corneal endothelium in all 10 eyes. It is not understood how the presence of silicone oil in the anterior chamber is implicated in the pathogenesis of band keratopathy but disturbances in the nutritional exchange of the cornea may be responsible.

In conclusion, the excimer laser is now an established surgical instrument and has been 
used successfully to treat a variety of superficia corneal pathologies. ${ }^{71-16}$ Given the ease with which the ophthalmologist may perform the procedure, the patient acceptance of the treatment, and the encouraging results in our series of 97 patients, we consider excimer laser PTK to be a safe and effective outpatient treatment for band keratopathy.

The authors acknowledge the Iris Fund for the Prevention of Blindness for continued financial support in relation to both the purchase and maintenance of the laser and provision of research fellowships for the principal investigators, Mr David O'Brart and Mr David Gartry. The authors thank Mr John Dart of Moorfield Eye Hospital for providing a specimen for histopathology. They thank Mr Mark Partridge of the Drug Information Unit and M Nicholas Taub of the Department of Public Health Medicine, United Medical and Dental Schools, St Thomas's Hospital They also thank Sister Ann Welch and Mrs Judith Armstron for clinical assistance and Mrs June Kraft for her secretarial assistance.

1 Dixon J. Diseases of the eye. 1st ed. London: J Churchill. 1848:

2 Bowman W. Lectures on anatomy of the eye. London Longmans, 1849; Appendix: 117

3 Grant WM. New treatment for calcific corneal opacities. Arch Ophthalmol 1952; 48: 681-5.

4 Breinin GM, DeVoe AG. Chelation of calcium with edathami calcium-disodium in band keratopathy and corneal calcium affections. Arch Ophthalmol 1954; 52: 846-51.

5 Sisson RJ. Band keratitis. Am f Ophthalmol 1934; 17: 222-9.

6 Wood TO, Walker GG. Treatment of band keratopathy. Am Ophthalmol 1975; 80: 553 .

7 Gartry D, Kerr Muir M, Marshall J. Excimer laser treatmet of corneal surface pathology: a laboratory and clinical study. BrF Ophthalmol 1991; 75: 258-69.
8 Marshall J, Trokel S, Rothery S, Schubert H. An ultrastructural study of corneal incisions induced by an excimer structural study of corneal incisions induced by
laser at $193 \mathrm{~nm}$. Ophthalmology $1985 ; 92: 749-58$.

9 Marshall J, Trokel S, Rothery S, Krueger RR. A comparative study of corneal incisions induced by diamond and steel knives and two ultraviolet radiations from an excimer laser. BrF Ophthalmol 1986; 70: 482-501.

10 Puliafito CA, Steinert RF, Deutsch TF, Hillenkamp F, Dehm EJ, Alder CM. Excimer laser ablation of the cornea and lens: experimental studies. Ophthalmology 1985; 92: 741-8

11 Marshall J, Trokel S, Rothery S, Kreuger RR. Photoablative reprofiling of the cornea using an excimer laser: photorefractive keratectomy. Lasers in Ophthalmology 1986; 1 : 21 48.

12 Sher NA, Bowers RA, Zabel RW, Frantz JM, Eiferman RA, Brown DC, et al. Clinical use of the $193 \mathrm{~nm}$ excimer laser in the treatment of corneal scars. Arch Ophthalmol 1991; 109 .
491-8.

13 Stark WJ, Chamon W, Kamp MT, Enger CL, Rencs EV, Gottsh JD. Clinical follow-up of $193 \mathrm{~nm}$ ArF excimer las photokeratectomy. Ophthalmology 1992; 99: 805-12.

14 Fagerholm P, Fitzsimmons TD, Orndahl M, Ohman L, Tengroth B. Phototherapeutic keratectomy with the excimer laser: long-term follow-up results in 166 treated eyes. Refractive and Corneal Surgery 1993; 9: S76-S81.

15 Steinert RF, Puliafito CA Excimer laser phototherapeutic keratectomy for a corneal nodule. Refractive Corneal Surgery 1990; 6: 352 .

16 Lohmann CP, Fitzke FW, O'Brart DPS, Timberlake G, Kerr Muir MG, Marshall J. Corneal light scattering and visual performance in myopes: a comparison between spectacles, contact lenses and excimer laser photorefractive keratectomy. Am f Ophthalmol 1993; 115: 444-53.

17 Pouliquen Y. Ultrastructure of band keratopathy. Arch Ophthalmol (Paris) 1967; 27: 149-58.

18 Cursino JW, Fine BS. A histologic study of calcific and noncalcific band keratopathies. Am $\mathcal{F}$ Ophthalmol 1976; 82: 395-404.

19 Sliney DH, Krueger RR, Trokel SL, Rappaport KD. Photokeratitis from $193 \mathrm{~nm}$ argon-fluoride laser radiation. Photochemistry and Photobiology 1991; 53: 739-44.

20 Brazier DJ, Hitchings RA. Atypical band keratopathy following long-term pilocarpine treatment. Br f Ophthalmol 1989; 73: $294-6$. 